\title{
Editorial
}

\section{Reconciliación social: tecnología para la construcción de paz}

\author{
MÓNICA ALZATE ${ }^{1}$
}

${ }^{1} \mathrm{PhD}$ en Psicología Social, Investigadora del Laboratorio de Innovación Social, Instituto Tecnológico Metropolitano, Medellín-Colombia, monicaalzate@itm.edu.co

Colombia es reconocida internacionalmente dentro de la lista de países en conflicto armando, es el segundo conflicto más antiguo del planeta [1]. La larga duración de este conflicto y su intensidad han dejado cerca de un $20 \%$ de la población en condiciones de victimización [2]; se estima que la inversión del país para contener la violencia corresponde a un 30\% de su PIB [3], es decir, seis veces más de lo que se invierte en educación. Es enorme el daño que ha ocasionado la confrontación armada, sin embargo, el país se encuentra ante una nueva oportunidad derivada del acuerdo de paz entre la guerrilla de las Farc y el Gobierno de Juan Manuel Santos.

Colombia iniciará una nueva etapa en la que será necesario recomponer los vínculos sociales que se rompieron y recuperar los niveles de desarrollo que se vieron interrumpidos a causa del conflicto. Esta etapa forma parte de lo que en las ciencias sociales se denomina reconciliación, definida como un proceso que conduce a la finalización estable de un conflicto, se basa en dos tipos de cambios: en la naturaleza de la relación entre los adversarios; y en las necesidades, emociones y cogniciones de cada una de las partes implicadas en el conflicto [4].

Valga puntualizar que la reconciliación abarca no solo la relación entre las víctimas y los excombatientes, sino que involucra a todos los individuos y grupos de una sociedad que participaron de la historia de confrontación; de ahí el importante papel que deberán jugar la institucionalidad y la población civil para alcanzar una paz duradera. Según [5], la mitad de los acuerdos de paz en el mundo fracasan dentro de los 5 años y no logran prevenir la reincidencia de la guerra. Esto hace indispensable una rápida y eficiente actuación para la (re)construcción de los lazos sociales y para el restablecimiento del equilibrio democrático. Pero, ¿cómo alcanzar esa eficiencia y eficacia? Quizá sea en la tecnología en donde se encuentre parte de la repuesta. Ese será el objetivo de este escrito, analizar el papel de la tecnología dentro del escenario del posacuerdo colombiano.

El uso de tecnología en las etapas de posconflicto ha sido recurrente tras la culminación de la violencia armada, especialmente en el continente africano, esto se ha atribuido a que la tecnología da un rápido retorno y mejoramiento de la competitividad de los países [6]. Según un informe presentado al Banco Mundial en 2014 cerca de cuarenta países han utilizado el apoyo de Tecnologías de la Información y de la Comunicación (TIC) para sus procesos de justicia transicional, como fue el caso de la Comisión de la Verdad y la Reconciliación de Sur- África [7]. La tecnología es de gran utilidad para implicar a la ciudadanía, las empresas y la sociedad civil organizada en las nuevas políticas públicas de construcción de paz, en el caso colombiano la utilidad se verá reflejada en varios aspectos: permitirán coexistencia y desarrollo de confianza; facilitarán la protección y diversificación de la 
ciudadanía; promoverán un mayor alcance y difusión del conocimiento; y producirán desarrollo económico sostenible e inversión. A continuación, comentaremos sobre cada uno.

\section{COEXISTENCIA Y CONSTRUCCIÓN DE CONFIANZA}

Mukashema y Mullet [8] plantean la existencia de dos componentes psicológicos que son fundamentales para la reconciliación social, la coexistencia y la construcción de confianza. Estos dos procesos requieren que los individuos y grupos desarrollen habilidades para contenerse en presencia de aquel que fue su adversario, además, les permite a las partes sentir que el opositor no les hará daño. Algunas de las intervenciones psicosociales que buscan desarrollar sentimientos de coexistencia y de confianza se basan en la Hipótesis de Contacto de Allport [9], consisten en propiciar interacciones controladas con algún miembro del grupo oponente; las interacciones a las que hacía referencia Allport eran reales, pero en estos tiempos bien podrían ser recreadas en escenarios virtuales, los desarrollos de la Hipótesis de Contacto se ponen en práctica a través de la forma que adoptan los mensajes institucionales en medios de comunicación y redes sociales.

El propósito de las intervenciones basadas en el trabajo de Allport es que se personalice a los miembros de un grupo opositor y que se evite otorgarles a los individuos particulares los atributos negativos que se asocian al grupo como un todo [10]. En el caso colombiano, se esperaría que en lugar de darle a una persona atributos por ser "guerrillero" o "paramilitar", se le evalúe por ser Juan, María o Pedro, es decir, un conciudadano con emociones, valores y características propias que no son homologables a las del grupo social. El uso de tecnología para presentar las historias de vida de los excombatientes facilitará ese proceso de personalización y promoverá la coexistencia.

Otra estrategia de intervención basada en el contacto y que se facilitará a través del uso de TIC es la propuesta por Petigrew [11], el autor propone difuminar las amenazas intergrupales por medio de disminuir la competición y enfatizar en los objetivos compartidos, esto promoverá el desarrollo de confianza entre las partes.

Un ejemplo de cómo este proceso puede darse a través de la transmisión de una noticia, fue publicado en el periódico $E l$ Colombiano. La Cancillería Colombiana informó que en Briceño (Antioquia) se avanzaba hacia el desminado, en la noticia se informó que participaban miembros de las Farc, y que se llevarán a cabo actividades complementarias que beneficiarán a la población, tales como la construcción del puente en la quebrada El Pescado, la biblioteca en Pueblo Nuevo, el proyecto de telemedicina en el casco urbano de Briceño y la capacitación de técnicos en salud pública. Además, se informó que ya se encuentran ejecutados los proyectos de potabilización de agua para las escuelas de cuatro veredas; la construcción de la biblioteca de Briceño y los quioscos de Vive Digital en Orejón y Pueblo Nuevo.

Esta noticia de La Cancillería sirve como facilitadora para la construcción de confianza hacia los miembros de las Farc, porque evidencia colaboración en lugar de competición y el interés por alcanzar unos objetivos compartidos que redundan en el beneficio de la comunidad. A través de esta noticia también se muestra la implementación de tecnología en programas como la telemedicina, los Quioscos de Vive Digital o la potabilización de agua. Valga mencionar que el propio desarrollo de dichos programas sirve como medio para facilitar la coexistencia y la construcción de confianza. 


\section{PROTECCIÓN Y DIVERSIFICACIÓN DE PARTICIPANTES}

Otra de las dimensiones que conforma la concepción de reconciliación social es la que se denomina reconciliación instrumental, es decir, la disposición hacia la tolerancia democrática. La reconciliación instrumental permite admitir que quien antes fuera un adversario ahora goza de los mismos derechos cívicos y políticos que tiene cualquier ciudadano [12]. Para que la reconciliación instrumental se alcance es indispensable fortalecer a las instituciones democráticas, pues solo de esta manera la población ejercerá una ciudadanía participativa en la que defienda sus derechos y asuma sus deberes para la cogobernanza del país. El fortalecimiento de la democracia implica que las instituciones sean capaces de proteger los derechos y la participación activa de los ciudadanos.

En cuanto al uso de tecnología para proteger a los ciudadanos y promover su participación, se encuentra un ejemplo en el conflicto de Liberia, en este país se utilizó una página web para la denuncia de violaciones a los Derechos Humanos, las denuncias eran anónimas y los servicios multimedia consiguieron recopilar narraciones que, entre otras cuestiones, facilitaron el encuentro entre las familias que se habían separado [13].

El uso de internet también ha permitido la organización de la sociedad civil, este es el caso de la masiva convocatoria que desató la denominada Primavera Árabe, o de las múltiples movilizaciones colectivas derivadas de la crisis política y económica que ha sacudido al planeta en la última década [14]. En el contexto colombiano, durante la realización de la mesa de conversaciones de la Habana se recibieron vía Web cerca de 11000 propuestas de la ciudadanía [15], permitiéndose así la visibilización de personas heterogéneas y

\footnotetext{
2 Ver www.mesadeconversaciones.com.co, 2016
}

que posiblemente por otro medio habrían visto restringida su participación. ${ }^{2}$

\section{MAYOR ALCANCE Y DIFUSIÓN DEL CONOCIMIENTO}

Tal y como ya se ha mencionado, el conflicto colombiano ha dejado cerca de ocho millones de habitantes en condición de víctima, así que generar información sobre las rutas de atención, el restablecimiento de sus derechos o la sistematización de la información podrá hacerse a través del uso de tecnologías digitales y sociales, tales como los Quioscos de Vive Digital [16], o como el índice de riesgo de victimización [17], o como los App para niños, niñas y adolescentes víctimas.

Otra tecnología social utilizada en países desarrollados y que busca disminuir los conflictos interpersonales es la ODR (Online Dispute Resolution) desarrollada por [18]; la ODR permite hacer una negociación directa entre dos partes en disputa. Poner la tecnología social al servicio de la paz se viene dando también desde enfoques societales, Collier ha trabajado en la búsqueda de indicadores demográficos que disminuyan la probabilidad de entrar en un conflicto, uno de sus resultados es que las poblaciones más educadas reducen la probabilidad de entrar en confrontación armada, cada año adicional de educación para la población general resulta en una reducción del 20 por ciento en la probabilidad de entrar en una guerra civil [19]. En este sentido y como un dato preocupante para el país, las Farc han informado que cerca del 80 por ciento de sus miembros son analfabetas, todas estas personas deberán alcanzar un nivel mínimo de educación y la tecnología será una herramienta ideal para facilitarla.

No solo la educación formal es importante para evitar la confrontación armada, también lo es la educación para la 
paz, esta es una de las grandes áreas que Naciones Unidas propone para prevenir la violencia. A través de esta se socializan las otras siete grandes áreas que contribuyen al mejoramiento de los indicadores nacionales de construcción de paz, entre otras: la igualdad entre hombres y mujeres; la importancia del libre flujo de la información; el fomento de la participación democrática; el desarrollo económico sostenible, etc. [20]. A este último indicador dedicaremos el siguiente apartado.

\section{DESARROLLO ECONÓMICO SOSTENIBLE E INVERSIÓN}

El desarrollo económico sostenible que debe llevarse a cabo para la construcción de paz está estrechamente relacionado con la creación de empresas de base tecnológica, pues estas generan altos niveles de riqueza para los países [6]. Según [21], las tecnologías que promueven las sociedades pacíficas incluyen el uso de la agricultura, la construcción y el transporte. En este sentido, desarrollar una empresa que a través de tecnología avanzada construya infraestructuras viales en las regiones más olvidadas de Colombia contribuirá a una mejor comercialización de los productos agrícolas y generará mejores ingresos para los cultivadores. Se espera que el desarrollo económico esté a su vez acompañado de sostenibilidad medioambiental, así que cabría esperar que tener unas mejores carreteras repercuta en una menor huella de carbono por la reducción en los tiempos de desplazamiento.

Durante las etapas de posconflicto, los países buscarán fomentar la inversión nacional y extranjera con el fin de poner en marcha ese desarrollo económico sostenible del que venimos hablando. En el caso colombiano, esto será particularmente interesante en aquellas regiones que dejarán de estar controladas por los grupos guerrilleros, pues se abrirán oportunidades de inversión pública y privada en sectores que antes no la recibían, es decir que se favorecerá la penetración de más y mejor tecnología al servicio de los ciudadanos. Los informes internacionales sostienen que la construcción de paz precisa hacer una distribución equitativa de recursos y del buen funcionamiento del gobierno, esto implica pensar en el bien común y por tanto en invertir en tecnología que redunde en la atención de necesidades básicas como la sanidad, la educación, la vivienda y los servicios públicos de calidad ${ }^{3}$.

Hasta el momento se ha mencionado la forma en que la tecnología contribuye de forma positiva a la construcción de paz, sin embargo, existe otra cara de la moneda. La tecnología también cuenta con el poder de dañar profundamente a las sociedades posconflictuales, pues permite la rápida destrucción de lazos sociales a través de la manipulación de la agenda pública en favor de los intereses de unos pocos, o una mayor expansión de otros tipos de violencia social, o la creación de una hipersensibilidad a las amenazas del posconflicto. También puede promover un rápido y desordenado desarrollo económico basado en la sobre explotación de los recursos naturales y sociales. Todos estos impactos negativos de la tecnología en la etapa del posacuerdo se verán moderados si se pone la tecnología al servicio de la sociedad y de la construcción de paz y no de la satisfacción de intereses particulares.

Para concluir, baste subrayar que la culminación de los conflictos bélicos es una oportunidad para que las naciones reorienten la forma en que quieren construir una nueva comunidad, hacerlo de la mano de la tecnología pondrá eficiencia y valor a esa reconstrucción social.

${ }^{3}$ Institute for Economics Peace, 2016 


\section{REFERENCIAS}

[1] A. B. Peiró, V. F. Armengol, I. N. Milián, J. M. R. Aspa, J. U. García, P. U. Arestizábal, A. V. Ariño, and M. V. Ariño, Alerta 2016! Informe sobre conflictos, derechos humanos $y$ construcción de paz, 1st ed. Barcelona: Ulzama, S.L., 2016.

[2] U. de Víctimas, "Solicitud de inscripción en el registro único de víctimas." Bogotá D.C., Colombia, 2017. Recuperado de http://www.unidadvictimas.gov.co/

[3] Institute for Economics and Peace (IEP), "Global Peace Index 2016," Sydney, New York, Brussels and Mexico City, 2016. Recuperado http://economicsandpeace.org/wpcontent/uploads/2016/06/GPI-2016Report_2.pdf

[4] A. Nadler, T. E. Malloy, and J. D. Fisher, The Social Psychology of Intergroup Reconciliation. Oxford University Press, 2008.

[5] Virginia M. Bouvier, Colombia La construcción de la paz en tiempos de guerra, 1st ed. Bogotá D.C., Colombia: Editorial Universidad del Rosario, 2014

[6] V. M. Tim Kelly, S. Raja, Christine, and Q. and M. Zhen-Wei Williams, "What role should governments play in broadband development?, in Dev/OECD workshop on "Policy Coherence in ICT for Development," 2009, p. 10. Recuperado de http://www.oecd.org/ict/4d/43631862.pdf

[7] D. Souter and T. Kelly, The Role of Information and Communication Technologies in Postconflict Reconstruction, World Bank. Washington, DC: The World Bank, 2013.

[8] I. Mukashema and E. Mullet, "Reconciliation Sentiment Among Victims of Genocide in Rwanda: Conceptualizations, and Relationships with Mental Health," Soc. Indic. Res., vol. 99, no. 1, pp. 25-39, Oct. 2010.

[9] G. W. Allport, The Nature of Prejudice, 1st ed. Massachusetts: Addison-Wesley Publishing Company, 1954.

[10] M. B. Brewer and N. Miller, "Beyond the Contact Hypothesis: Theoretical Perspectives on Desegregation," in Groups in Contact, 1ST ed., ACADEMIC PRESS INC., Ed. Orlando: Elsevier, 1984, pp. 281-302.
[11] T. F. Pettigrew, "Applying Social Psychology to International Social Issues," J. Soc. Issues, vol. 54, no. 4, pp. 663-675, Jan. 1998.

[12] M. Alzate, J. M. Sabucedo, and M. Durán, "Antecedents of the attitude towards intergroup reconciliation in a setting of armed conflict," Psicothema, vol. 25, no. 1, pp. 61-66, 2013.

[13] T. N. Smyth, J. Etherton, and M. L. Best, "MOSES: Exploring new ground in media and post-conflict reconciliation," in Proceedings of the 28th international conference on Human factors in computing systems - CHI '10, 2010, pp. 1059-1069.

[14] E. Anduiza, C. Cristancho, and J. M. Sabucedo, "Mobilization through online social networks: the political protest of the indignados in Spain," Information, Commun. Soc., vol. 17, no. 6, pp. 750-764, Jul. 2014.

[15] Mesa de Conversaciones de la Habana, "Propuestas a la mesa de conversaciones," Propuestas a la mesa de conversaciones, 2016. Recuperado

de https://www.mesadeconversaciones.com.co/.

[16] J. J. B. García, R. A. González, and L. O. Bello, "Una estrategia para la apropiación de las TIC en la reconciliación de las víctimas del conflicto armado colombiano.," TRILOGÍA. Ciencia, Tecnol. y Soc., vol. 8, no. 14, pp. 5364, 2016

[17] U. de Víctimas, "Indice de riesgo de victimización 2010-2012," Bogotá D.C., Colombia, 2015.

[18] M. H. Conley Tyler and M. W. McPherson, "Online Dispute Resolution and Family Disputes," J. Fam. Stud., vol. 12, no. 2, pp. 165-183, Nov. 2006.

[19] P. Collier, "Economic causes of civil conflict and their implications for policy," Washington D.C., 1, 2007.

[20] M. Alzate, J. M. Sabucedo, and M. y Durán, "The transformation of a Socio-Political Conflict: Building a Culture of Peace in Colombia," in Psychosocial Approaches to Peace-Building in Colombia, 1st ed., vol. 25, S. Sacipa-Rodriguez and M. Montero, Eds. Cham: Springer International Publishing, 2014, pp. 137-151.

[21] Brian Martin, "Technology, violence and peace," Encyclopedia of Violence, Peace and Conflict. University of Wollongong, pp. 2044$2055,2010$. 